\title{
The importance of an intraoperative revision of all parathyroid glands, which may reveal additional pathologic changes and lead to an operation extension
}

Nývltová M., Drbalová K., Krejčí P., Zavoral M.

Department of Internal Medicine of First Faculty of Medicine of Charles University and Military University Hospital, Prague, Czech Republic

We present two case reports of patients who were diagnosed with primary hyperparathyroidism based on a parathyroid gland adenoma (PTA). The pathological parathyroid tissue was precisely localised by ultrasonography and scintigraphy before the surgery. In both cases all four parathyroid glands were revised by the surgeon which resulted in an operation extension.

\section{First case report :}

The first patient (male, 72 years old) was diagnosed with hyperparathyroidism in 10/2012. Ultrasonography showed a goiter with a solitary cystic nodule in the right lobe (picture 1, picture 2). Scintigraphy (Tc-MIBI) didn't show any hyperfunctional parathyroid tissue (picture 3). In the cystic fluid from the nodule (acquired by FNAC) we measured very high concentration of PTH (53 $000 \mathrm{pmol} / \mathrm{l})$.
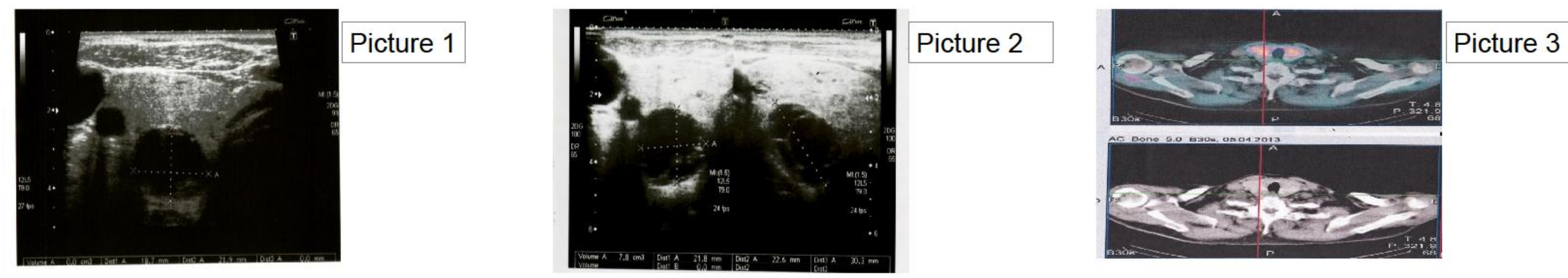

The patient was recommended for a total thyroidectomy and extirpation of the right inferior PTA.

From the operation protocol and histology results:

1/ extirpation of an obvious adenoma of a left superior parathyroid gland !!! 16x9x7 mm, which was confirmed histologically

$2 /$ removement of a prominence of thymus with a suspicious parathyroid tisuue- histologically normal parythyroid gland

$3 /$ total thyroidectomy -histologically multinodular goiter.

The pathologist surprisingly didn't describe any parathyroid tissue within or next to the right lobe of the thyroid gland!

\section{Second case report :}

The second patient (female, 77 years old) was diagnosed with a hyperparathyroidism in 6/2015.

Ultrasonography showed a small thyroid gland with one isoechoic nodule in the right lobe of the thyroid gland and two suspicious hypoechoic nodules under the left lobe (picture 4). Scintigraphy revealed two PTAs - left superior (picture 5) and right inferior (picture 6).

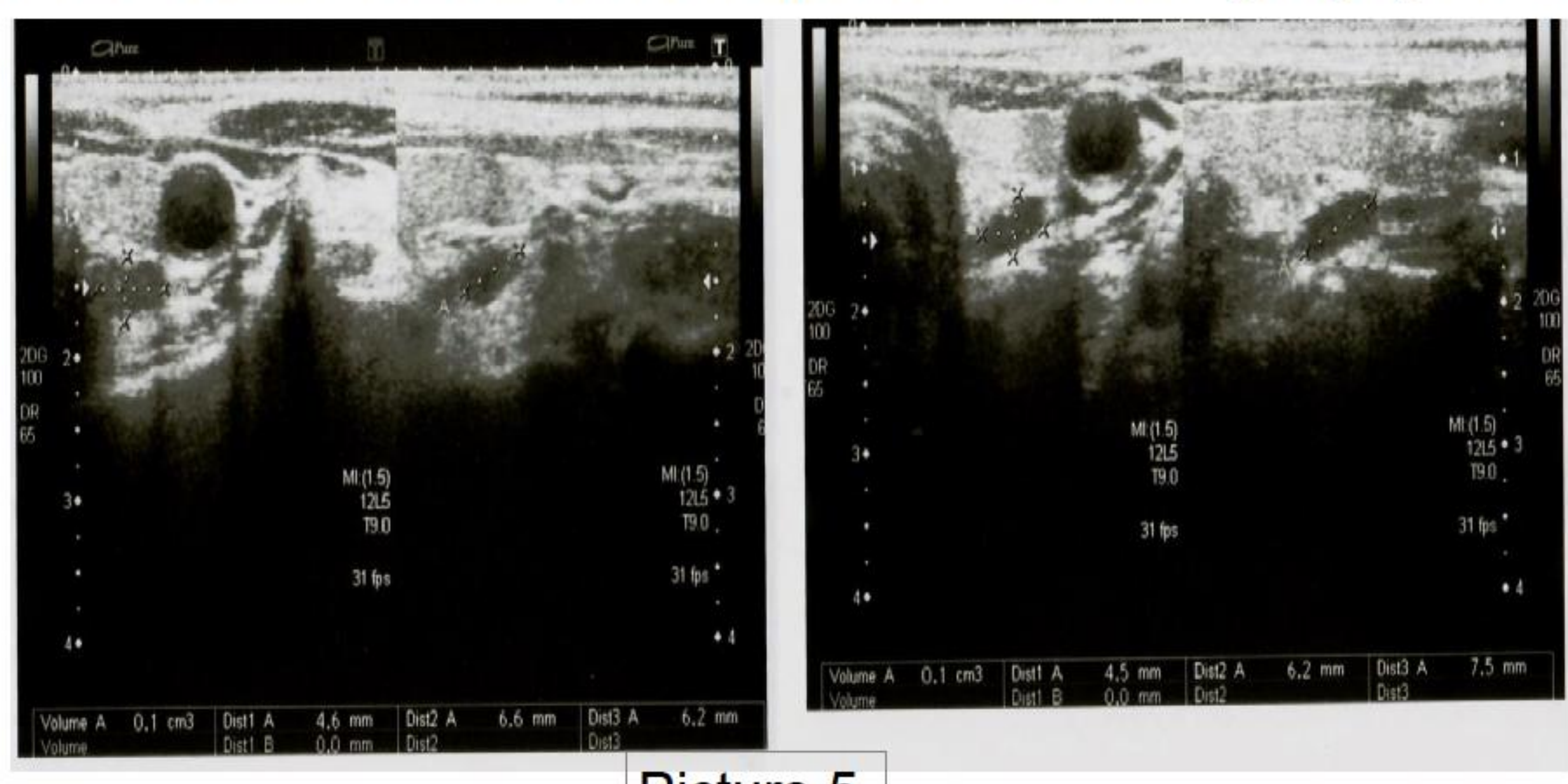

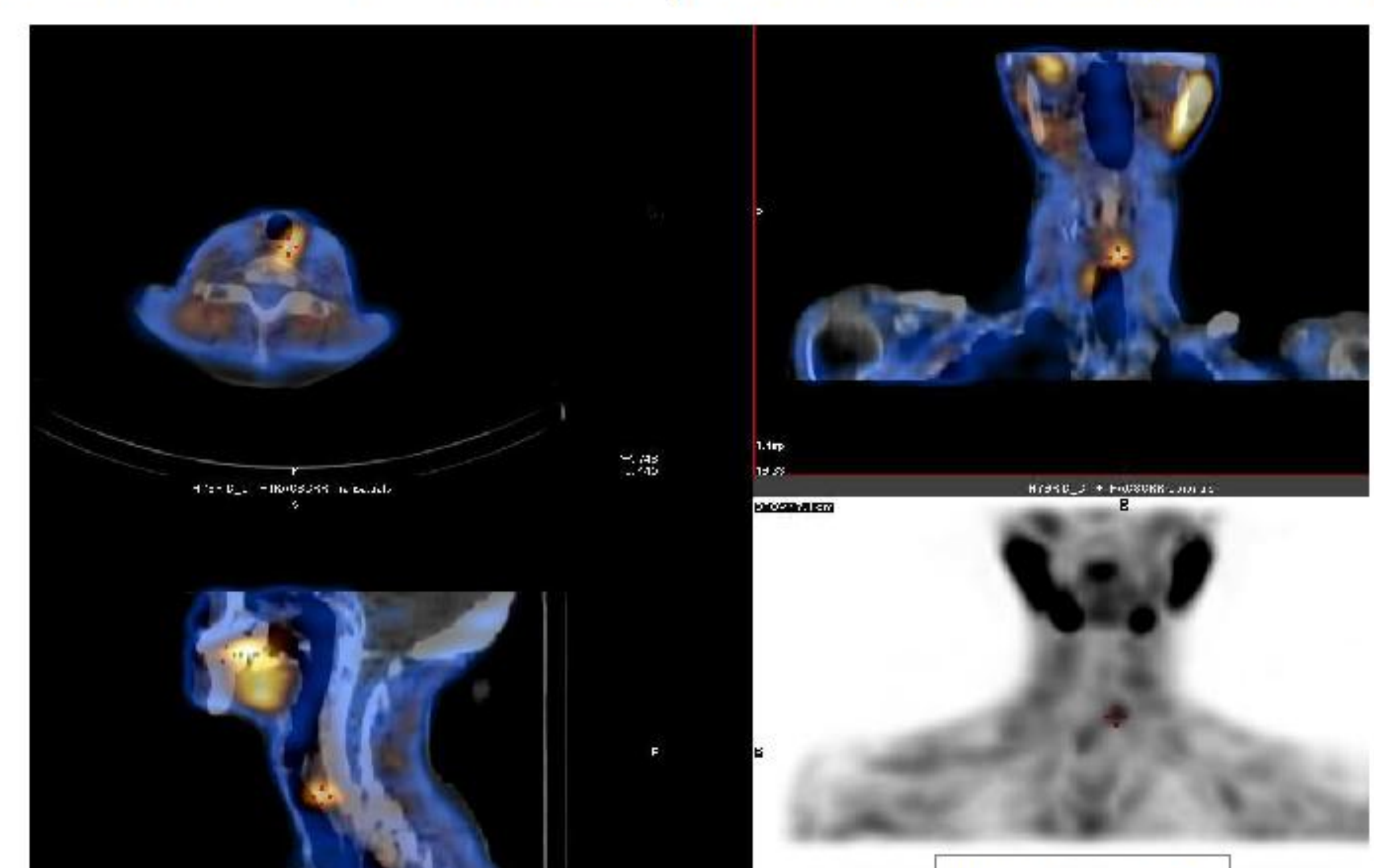

Picture 6

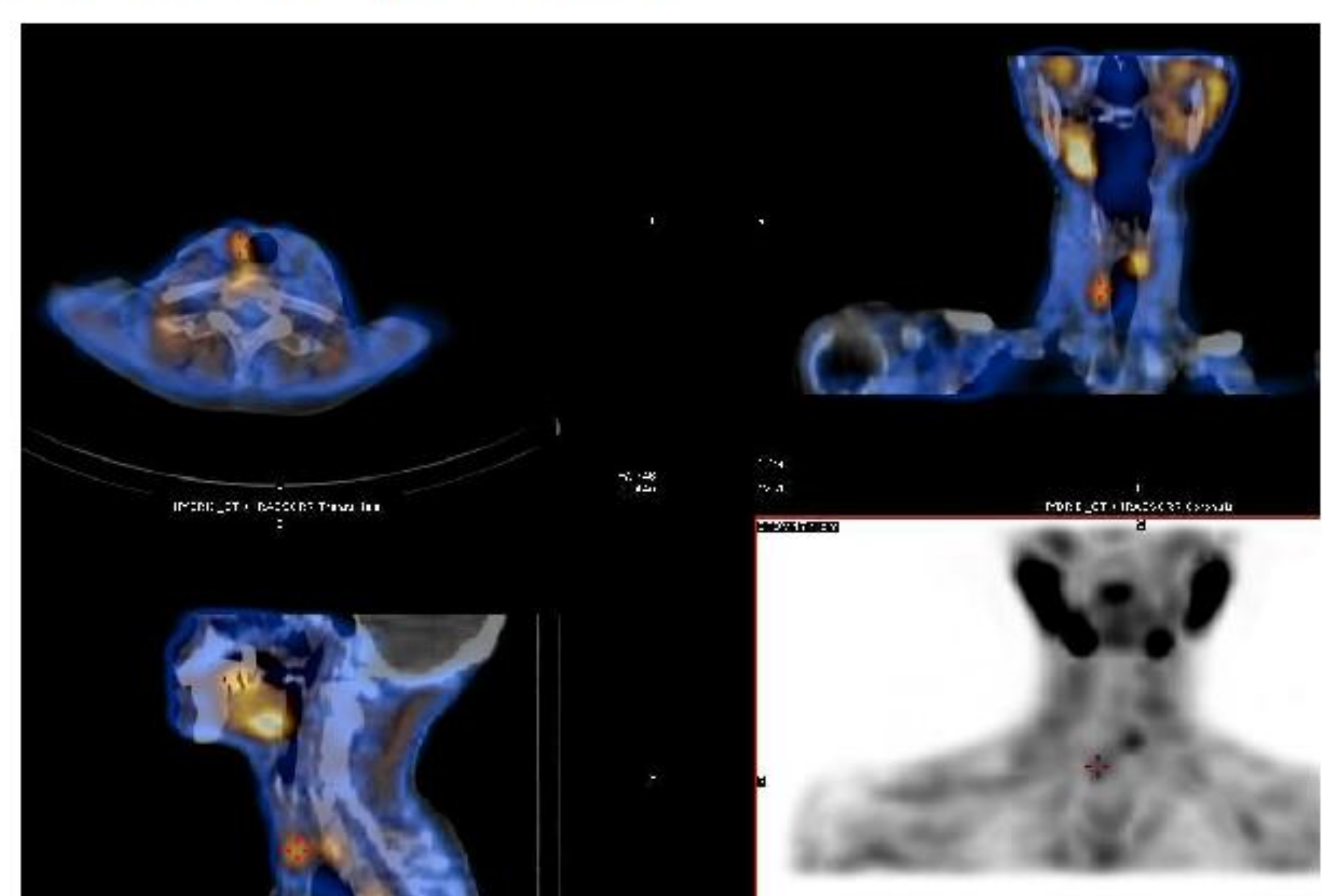

Picture 7

The patient was recommended for a right-side hemityroidectomy and extirpation of two PTAs.

From the operation protocol and histology results:

1/ definitely enlarged left superior parythyroid gland $(15 \times 10 \times 4 \mathrm{~mm})-$ histologically PTA as anticipated.

$2 /$ definitely enlarged right superior parathyroid gland $(15 \times 10 \times 4 \mathrm{~mm})-$ histologically hyperplasia of main parathyroid cells

$3 /$ partial resection of a slightly enlarged right inferior parathyroid gland ( $3 \times 3 \mathrm{~mm}$ left) - histologically normal parythyroid gland tissue!

In summary, the surgeon had doubts and decided not to remove the whole right inferior parathyroid gland and this decision was correct,

because from the resected tissue the pathologist reported normal parathyroid tissue! Furthermore, another PTA of the right superior parathyroid gland was extirpated.

\section{Conclusion :}

Those two cases highlight the important role of an intraoperative revision af all parathyroid glands by the surgeon.

In the first case there was high PTH detected in the cystic nodule in the right lobe of the thyroid gland (or under it), but neither surgeon nor pathologist found PTA. Despite the scintigraphy didn't show any hyperfunctional tissue on the left side of the neck, the surgeon had removed and the pathologist confirmed another PTA of a left superior parathyroid gland!

In the second case the patient was recommended for surgery with two suspicious PTAs of a left superior and right inferior parathyroid gland. Left superior PTA was obvious and confirmed histologically. But the surgeon had doubts about the right inferior PTA. Therefore he made only a partial resection and this was a good decision, because histologically it was normal parathyroid tissue! Moreover, during the peroperative revision there was found one more PTA of a right superior parathyroid gland.

In both cases the surgeon's approach was beneficial for the patient and both operation procedures were therefore curative. 